\title{
Biological activity of cytotoxic dendritic cells cocultured with cytokine-induced killer cells and their effect on acute leukemia cells
}

\author{
X.Y. Cheng and J.L. Li \\ Department of Analgesia, The People's Hospital, Weifang, Shandong, China \\ Corresponding author: J.L. Li \\ E-mail: wutq12@126.com
}

Genet. Mol. Res. 14 (4): 13208-13214 (2015)

Received April 23, 2015

Accepted August 19, 2015

Published October 26, 2015

DOI http://dx.doi.org/10.4238/2015.October.26.17

ABSTRACT. We cocultured cytokine-induced killer (CIK) cells with dendritic cells (DCs) in vitro and investigated their proliferation, immunophenotype changes, secretory cytokine levels, and their antitumor effects on acute myeloid leukemia (AML) cells. DCs and CIK cells were acquired from healthy human peripheral blood mononuclear cells and cocultured as an experimental group, while CIK cells were cultured alone as a control group. Cell numbers were counted by trypan blue staining, cytotoxic activity was measured by a 3-(4,5-dimethylthiazol-2-yl)-2,5-diphenyltetrazolium bromide assay, cell phenotypes were detected by flow cytometry, and secreted levels of INF- $\mathrm{Y}$ and IL-12 were determined by enzyme-linked immunosorbent assay. The proliferation activity in the experimental group was noticeably higher than in the control group $(P<0.05)$. Under the same conditions, the ratio of $\mathrm{CD}^{+} \mathrm{CD} 56^{+}$and $\mathrm{CD} 3^{+} \mathrm{CD} 8^{+}$double-positive CIK cells was significantly elevated when cocultured with DCs $(P<0.05)$. Compared with the control group, the experimental group had significantly higher levels of secreted INF- $Y$ and IL-12 in the supernatants after 3 days $(P<0.01$ and $P<0.05$, respectively). The antitumor effect of DC-CIK cells against leukemia cells was much higher than that of CIK cells at an effector-target ratio ranging from $2.5: 1$ to $20: 1(P<0.05)$, and this effect 
was positively related to the effector-target ratio. The proliferation activity, level of secretory cytokines, and antitumor effect against AML cells of DC-CIK cells were significantly higher than in CIK cells. This study provides a theoretical and experimental basis for clinical immunotherapy using DC-CIK cells.

Key words: Cytokine-induced killer cells; Dendritic cells; DC-CIK; Acute myeloid leukemia cells; Cytotoxic effect

\section{INTRODUCTION}

Hematological malignancies are malignant cancers that affect the blood, bone marrow, and lymph nodes (Maciejewski and Haferlach, 2012). Traditional treatments for hematological malignancy comprise chemotherapy, radiotherapy, immunotherapy, and bone marrow transplant. Chemotherapy and radiotherapy are common approaches to tumor treatment. However, healthy cells are also damaged during such treatments, which eventually leads to a weaker immune system. This diminishes patients' ability to counteract the tumor cells. Moreover, bone marrow transplant involves a high degree of risk. Therefore, immunotherapy is attracting increasing attention (Stamatovic et al., 2011).

Dendritic cells (DCs) are the main antigen-presenting cells (APCs) in the human body; they can present antigens to $\mathrm{T}$ lymphocytes and induce cytotoxic $\mathrm{T}$ cell responses in vivo and in vitro. Of all the APCs, only DCs have the ability to induce primary immune responses in resting naive $\mathrm{T}$ lymphocytes. DCs not only induce $\mathrm{T}$ cell activation but also play important roles in the immune tolerance and functions of B cells. Cytokine-induced killer (CIK) cells are a type of common antitumor immunocompetent cells. They exhibit strong proliferation capacity and high antitumor activity in vitro, and they are particularly effective against multidrug-resistant tumor cells (Yamaguchi et al., 2003). Previous studies have shown that when DCs are cocultured with CIK cells in vitro, they can promote the proliferation of CIK cells and enhance their cytotoxic effects against tumor cells (Marten et al., 2001b). Further, when DCs and CIK cells are cocultured with some antigen peptides, the ability of the DC-CIK combination to destroy some tumor cells lacking specific antigens is enhanced. Hence, the combined use of CIKs and DCs in the treatment of malignant tumors provides tumor patients relief from the partial immune anergy of $\mathrm{T}$ cells, thereby enhancing the antitumor effect. Indeed, DC-CIK immunotherapy has already been applied to the treatment of many types of cancers such as melanoma, prostate cancer, and renal cell carcinoma (Fujii et al., 1999; Rieser et al., 1999; Burch et al., 2000; Geiger et al., 2000; Toungouz et al., 2001).

The combined use of CIKs and DCs has a significant antitumor effect, but it is not known whether it is effective against acute leukemia. In this study, we adopted combinations of several cytokines to induce DCs and CIKs from peripheral blood and then cocultured the cells. The proliferative activities, phenotypes, and the cytotoxic effect against acute myeloid leukemia (AML) cells of the DCCIK cells were then examined. Our objective was to provide an experimental basis for the future clinical application of the DC-CIK combination in immunotherapy against hematological malignancy.

\section{MATERIAL AND METHODS}

\section{Main reagents}

Roswell Park Memorial Institute (RPMI) 1640 culture medium was bought from Gibco (Grand Island, NY, USA). Recombinant rhGM-CSF and rhIL-2 were purchased from Shanghai 
Huaxin Biological Technology Co., Ltd. Recombinant rhIL-4, rhIFN-y, and rhTNF- $\alpha$ were provided by Shanghai Chong Heng Biotechnology Co., Ltd. $\mathrm{CD} 3^{+}, \mathrm{CD}^{+} \mathrm{CD} 4^{+}, \mathrm{CD} 3^{+} \mathrm{CD} 8^{+}$, and $\mathrm{CD} 3^{+} \mathrm{CD} 56^{+}$ antibodies labeled with fluorescence molecules for flow cytometry assays and an enzyme-linked immunosorbent assay (ELISA) kit for detecting cytokines (IL- 12, IFN- $\gamma$ ) were obtained from R\&D Systems. Mouse anti-human CDla, CD14, and HLA-DR, and fluorescein isothiocyanate-labeled rabbit anti-rat conjugated second antibody were purchased from the Chinese Academy of Medical Sciences, Tianjin Institute of Hematology.

\section{Sources of target cells}

Leukemia cell samples were obtained from patients at the department of hematology in our hospital. The target cells were acquired from leukemia patients whose blood samples had been subjected to diagnostic criteria after performing comprehensive analyses of the clinical symptoms, signs, and peripheral blood cytology. The morphological characteristics of the bone marrow cells, the bone marrow biopsy results, and the cellular immunology and molecular cell biology detection results were also scrutinized. Written informed consent was obtained from the patients and approved by the local institutional ethics committee.

\section{Culture and amplification of CIK cells}

Peripheral blood was collected from healthy blood donors and mononuclear cells were separated by centrifugation using a lymphocyte separating medium. All the samples were incubated in a humidified incubator at $37^{\circ} \mathrm{C}$ in $5 \% \mathrm{CO}_{2}$ for $2 \mathrm{~h}$; then, non-adherent cells were collected and the cell density was adjusted to $3 \times 10^{6} / \mathrm{mL}$ with RPMI 1640 complete medium before culturing on a 24-well plate. rhIFN-y $(10 \mathrm{U} / \mathrm{mL})$ was added to the culture, which was incubated at $37^{\circ} \mathrm{C}$ in $5 \%$ $\mathrm{CO}_{2}$ for $24 \mathrm{~h}$. Next, CD3 monoclonal antibody $(50 \mathrm{ng} / \mathrm{mL})$ and $\mathrm{rhlL}-2(300 \mathrm{U} / \mathrm{mL})$ were added, and the medium was replaced with fresh medium comprising $300 \mathrm{U} / \mathrm{mL}$ rhlL-2 every 3 days. Human experiments were performed in accordance with the ethical standards provided by the responsible committee of the institution and in accordance with the Declaration of Helsinki (as revised in Edinburgh 2000). All human subjects gave their informed consent.

\section{In vitro culture of DCs}

The cell density of adherent cells was adjusted to $1 \times 10^{6} / \mathrm{mL}$ with medium containing 1000 $\mathrm{U} / \mathrm{mL}$ rhGM-CSF and $500 \mathrm{U} / \mathrm{mL}$ rhIL4. The cells were then cultured on 24 -well culture plate at $37^{\circ} \mathrm{C}$ in $5 \% \mathrm{CO}_{2}$, and half the medium was changed for fresh medium containing cytokines every other day. The cytokine rhTNF- $\alpha(50 \mathrm{U} / \mathrm{mL})$ was added to the culture medium to induce dendritic cell maturation for $72 \mathrm{~h}$ before harvesting the cells for subsequent experiments.

\section{DC-CIK cells coculture}

DCs were collected and counted; then, they were co-cultured with CIKs at a ratio of 1:5 with CIK cell culture medium. The initial cell density was $5 \times 10^{5} \mathrm{cells} / \mathrm{mL}$, and the medium was replaced with fresh CIK cell culture medium every 2 or 3 days. 


\section{Analysis of cytotoxicity activity}

The frozen acute leukemia cells were resuscitated and used as the target cells. CIK cells and the cocultured DC-CIK cells were used as the effector cells. The effector and target cells were inoculated onto the culture plate at ratios of 2.5:1, 5:1, 10:1, and 20:1. Some wells containing only effector cells or target cells were set as controls. There were three parallel wells for each effectortarget ratio. The culture plates were placed in a humidified incubator at $37^{\circ} \mathrm{C}$ in $5 \% \mathrm{CO}_{2}$ for 48 h. Then, $20 \mu \mathrm{L}$ 3-(4,5-dimethylthiazol-2-yl)-2,5-diphenyltetrazolium bromide (MTT) was added to each well and incubated for another $4 \mathrm{~h}$ before adding $100 \mu \mathrm{L}$ dimethyl sulfoxide to each sample and subsequently stirring sufficiently. Next, an enzyme mark instrument was applied to measure the absorbance value of the liquid culture medium at $570 \mathrm{~nm}$ for cytotoxicity rate analysis.

Cytotoxicity rate $(\%)=[1$ - (effector-target group A - effector group A)/target cell A $] \times 100 \%$.

\section{Analysis of immunophenotypes}

Flow cytometry was applied to investigate the immunophenotypes of the CIK and DC-CIK cells, and the immunophenotypes of the DCs were detected by an indirect immunofluorescence assay.

\section{Detection of cytokines}

A double sandwich ELISA method was used to analyze the expression of IL-12 in the supernatants according to manufacturer instructions provided with the ELISA kit.

\section{Statistical analysis}

All data were analyzed using SPSS13.0 statistics software, and the results are shown as means \pm standard deviation (SD). Comparison between groups was conducted by the double $t$-test and $\mathrm{P}<0.05$ was considered statistically significant.

\section{RESULTS}

\section{Observation of proliferation in the CIK and DC-CIK cells}

No obvious differences were observed in the multiplication of DCs and CIK cells during the first 2 days of coculture. Afterwards, with the extension of culture time, the proliferation rate of the DC-CIK cells was significantly higher than that in the homologous ClK cells. Cell viability was determined using the trypan blue exclusion method. As shown in Table 1, the total cell number in the CIK control group increased by $28.7 \pm 4.95$ fold after 12 days and $45.63 \pm 4.59$ fold after 15 days, while in the DC-CIK experimental group, the increase was $42.7 \pm 4.89$ fold after 12 days and $63.56 \pm 3.76$ fold after 15 days. The results were analyzed and the difference between the two groups was statistically significant $(P<0.05)$.

\section{Detection of immunophenotypes in the CIK and DC-CIK cells}

Immunophenotype analysis of the CIKs indicated that, when cultured alone, the proportions of $\mathrm{CD}^{+} \mathrm{CD}^{+}$and $\mathrm{CD}^{+} \mathrm{CD} 56^{+}$increased gradually with increasing culture time. Under the same 
conditions, the proportions of both $\mathrm{CD} 3^{+} \mathrm{CD} 8^{+}$and $\mathrm{CD} 3^{+} \mathrm{CD} 56^{+}$double-positive cells in the $\mathrm{DC}-\mathrm{CIK}$ group was noticeably higher than in the control group when CIK cells were cultured alone $(P<$ 0.05) (Table 2).

Table 1. Relationship between cell proliferation rate and culture time (means $\pm s$ ).

\begin{tabular}{lcrr}
\hline Group & Number of cases & \multicolumn{2}{c}{ Multiplier } \\
\cline { 2 - 3 } & & $12 \mathrm{~d}$ & $15 \mathrm{~d}$ \\
\hline CIK & 20 & $28.7 \pm 4.95$ & $45.63 \pm 4.59$ \\
DC-CIK & 20 & $42.7 \pm 4.89^{*}$ & $63.56 \pm 3.76^{*}$ \\
\hline
\end{tabular}

CIK = cytokine-induced killer; $\mathrm{DC}=$ dendritic cells; $d=$ day, ${ }^{*} \mathrm{P}<0.05$.

Table 2. Phenotype changes in the CIK and DC-CIK cells (\%, means $\pm \mathrm{s})$.

\begin{tabular}{|c|c|c|c|c|c|}
\hline \multirow[t]{3}{*}{ Group } & \multicolumn{5}{|c|}{ Culture time } \\
\hline & \multirow[t]{2}{*}{ Control (MNC) } & \multicolumn{2}{|c|}{$3 d$} & \multicolumn{2}{|c|}{$6 \mathrm{~d}$} \\
\hline & & CIK & DC-CIK & CIK & DC-CIK \\
\hline CD3+ cells & $58.19 \pm 2.89$ & $63.48 \pm 3.57$ & $72.42 \pm 4.68$ & $78.24 \pm 3.78$ & $81.63 \pm 7.32$ \\
\hline CD3+CD4+ cells & $37.84 \pm 2.97$ & $18.58 \pm 2.04$ & $19.85 \pm 3.55$ & $20.42 \pm 2.26$ & $21.94 \pm 4.34$ \\
\hline CD3+CD8+ cells & $26.39 \pm 3.48$ & $39.86 \pm 3.58$ & $58.42 \pm 4.12^{*}$ & $63.56 \pm 5.45$ & $76.28 \pm 5.54^{*}$ \\
\hline CD3+CD56+ cells & $4.03 \pm 0.98$ & $23.42 \pm 5.17$ & $36.38 \pm 2.42^{*}$ & $35.21 \pm 2.65$ & $49.56 \pm 2.98^{*}$ \\
\hline
\end{tabular}

$\mathrm{CIK}=$ cytokine-induced killer; $\mathrm{DC}=$ dendritic cells; MNC = mononuclear cells; $\mathrm{d}=$ day, ${ }^{*} \mathrm{P}<0.05, \mathrm{DC}-\mathrm{CIK}$ vs CIK under the same conditions.

\section{Cytotoxic activity of the DC-CIK and CIK cells}

The analysis results showed that both CIK and DC-CIK cells powerfully inhibited the growth of various kinds of AML cell subtypes when the effector-target ratio was in the range 2.5:1 to 20:1. As can be seen from Table 3, the cytotoxic activity was obviously related to the effector-target ratio and increased with increase in the effector-target ratio. The cytotoxic activity of the DC-CIK cells against various kinds of AML cell subtypes was markedly higher than that of the CIKs under the same conditions $(P<0.05)$. However, there were no significant differences in the cytotoxic activity of CIK cells (or CD-CIK cells) on the different AML cell subtypes at the same effector-target ratio $(P>0.05)$.

Table 3. Cytotoxic activity of the CIK and DC-CIK cells when cultured with AML cells ( $\%$, means $\pm s$ ).

\begin{tabular}{|c|c|c|c|c|c|c|}
\hline \multirow[t]{2}{*}{ Group } & \multirow[t]{2}{*}{ Target cells } & \multirow[t]{2}{*}{ Number of cases } & \multicolumn{4}{|c|}{ Effect of effector-target ratio } \\
\hline & & & $2.5: 1$ & $5: 1$ & $10: 1$ & $20: 1$ \\
\hline \multirow[t]{4}{*}{ CIK } & AML-M1,2 & 16 & $11.27 \pm 5.35$ & $15.93 \pm 4.23$ & $21.83 \pm 3.84$ & $30.23 \pm 3.94$ \\
\hline & AML-M3 & 18 & $13.15 \pm 3.12$ & $18.21 \pm 2.01$ & $23.42 \pm 4.29$ & $33.09 \pm 5.21$ \\
\hline & AML-M4,5 & 20 & $16.02 \pm 1.98$ & $19.23 \pm 3.51$ & $26.32 \pm 3.52$ & $36.13 \pm 5.97$ \\
\hline & AML-M6 & 14 & $12.32 \pm 4.54$ & $16.83 \pm 3.92$ & $23.63 \pm 5.09$ & $30.41 \pm 3.33$ \\
\hline \multirow[t]{4}{*}{ DC-CIK } & AML-M1,2 & 16 & $18.43 \pm 4.24$ & $24.91 \pm 3.92$ & $31.58 \pm 2.09$ & $42.84 \pm 2.57$ \\
\hline & AML-M3 & 18 & $19.56 \pm 3.32$ & $28.03 \pm 2.91$ & $34.14 \pm 4.97$ & $45.82 \pm 3.97$ \\
\hline & AML-M4,5 & 20 & $23.06 \pm 3.65$ & $30.11 \pm 4.94$ & $36.19 \pm 5.24$ & $49.57 \pm 3.02$ \\
\hline & AML-M6 & 14 & $18.97 \pm 5.43$ & $26.91 \pm 3.57$ & $34.98 \pm 2.21$ & $41.57 \pm 3.07$ \\
\hline
\end{tabular}

$\mathrm{CIK}=$ cytokine-induced killer; $\mathrm{DC}=$ dendritic cells; $\mathrm{AML}=$ acute myeloid leukemia, $\mathrm{P}<0.05$, DC-CIK vs CIK under the same conditions 


\section{Measurement of cytokine levels}

The supernatants of the DC-CIK cells were collected for the measurement of IFN-Y and IL-12 cytokine expression levels after 3 days of coculture. The results showed that the levels of IFN-y and IL-12 in the DC-CIK group were markedly elevated compared with the CIK group $(\mathrm{P}<$ $0.05, \mathrm{P}<0.01$ ) (Table 4).

Table 4. Comparison of secreted cytokine levels between CIK and DC-CIK cells (means $\pm s$ ).

\begin{tabular}{lclr}
\hline Group & Number of cases & IFN-y $(\mathrm{pg} / \mathrm{mL})$ & $\mathrm{IL}-12(\mathrm{pg} / \mathrm{mL})$ \\
\hline CK & 16 & $246.64 \pm 84.16$ & $12.08 \pm 0.84$ \\
DC-CIK & 18 & $542.16 \pm 198.25^{*}$ & $72.48 \pm 10.09^{* *}$ \\
\hline CIK = cytokine-induced killer; DC $=$ dendritic cells, ${ }^{*} \mathrm{P}<0.05,{ }^{* *} \mathrm{P}<0.01$. &
\end{tabular}

\section{DISCUSSION}

ClK cells are a kind of cytotoxic T lymphocyte that can be induced by various cytokines such as IL-2 and IFN- $\gamma$, and also by the CD3 monoclonal antibody; they destroy various tumors and their cytotoxic capacity is not restricted by the major histocompatibility complex (Nagaraj et al., 2004). The homing process of CIKs takes place in the spleen and lymph nodes, and they can specifically localize to tumor tissues to exert a partial antineoplastic effect (Gritzapis et al., 2002). DCs, the most effective antigen-presenting cells in vivo, are distributed widely in different tissues of the body except for the testis and brain (van den Broeke et al., 2003). DCs take up exogenous antigens with the help of their receptors and can combine with class I major histocompatibility complex molecules on the surface of those antigens, thereby stimulating the activation of naive CD8 ${ }^{+} \mathrm{T}$ cells and CD4 ${ }^{+}$ T cells. Apart from inducing antigen-specific cytotoxic T lymphocytes, DCs can directly or indirectly influence the proliferation of B cells and activate humoral immune responses (Cao, 2003). To date, DC-based antitumor immunotherapy has been applied to the treatment of cancers like melanoma, prostate cancer, renal cell carcinoma, chronic myeloid leukemia, and solid tumors (Fujii et al., 1999; Rieser et al., 1999; Burch et al., 2000; Geiger et al., 2000; Toungouz et al., 2001). It has been reported that the secretion of cytokines by CIKs and DCs promotes their proliferation when DC cells are incubated together with CIK cells; the cytotoxic effects are enhanced and the innate and acquired immune activation of the organism is activated. Therefore, the combined application of CIKs possessing cytotoxic activity and DCs possessing the capability of presenting tumor antigens may enhance the antitumor effect, which has great potential for the treatment of malignant tumors.

Märten et al. (Marten et al., 2001a) found that coculturing ClK cells with homologous DCs greatly promoted the secretion of IL-12, thereby enhancing the cytotoxic activity of CIKs against tumor cells. In keeping with that finding, in our study the levels of IFN-y and IL-12 in the DC-CIK group were markedly elevated compared with the $\mathrm{CIK}$ group. $\mathrm{CD} 3^{+} \mathrm{CD} 8^{+}$and $\mathrm{CD} 3^{+} \mathrm{CD} 56^{+} \mathrm{T}$ cells are major members of the human immune system, and play an important role in killing tumor cells and virus-infected cells. In our study, compared with the CIK cells cultured alone, the proportion of $\mathrm{CD}^{+}{ }^{+} \mathrm{CD} 8^{+}$and $\mathrm{CD}^{+} \mathrm{CD} 56^{+}$double-positive cells in the CIKs cocultured with DCs was significantly higher under the same conditions, suggesting that the DC-CIK cells have a more powerful antitumor effect. Preclinical studies have shown that CIK cells in leukemia patients can kill autologous and xenogenous leukemia cells and clear residual leukemia cells (Linn and Hui, 2003). In our study, the AML cell subtypes (AML1 to AML6) acquired from leukemia patients were set as target cells, 
and the results showed that the cytotoxic activity of the DC-CIK cells against various kinds of AML cell subtypes was significantly higher than that of the CIK cells under the same conditions. Our research indicated that the co-cultured DC-CIK cells not only had a higher proliferation rate but also possessed strong cytotoxic activity against tumor cells such as AML cells.

Combined treatment with CIKs and DCs can greatly enhance the antitumor capacity of CIKs and improve the specificity of CIKs acting against target cells; it is expected to become the optimal option for the immunotherapy of hematological malignancy. This study suggests that the proliferative activity of DC-CIK cells and their cytotoxic activity against various kinds of AML cell subtypes are significantly higher than in CIKs under the same conditions, which provides a theoretical and experimental basis for the application of DC-CIK cells co-cultured in vitro to the immunotherapy of hematological malignancy.

\title{
Conflicts of interest
}

The authors declare no conflict of interest.

\section{ACKNOWLEDGMENTS}

\author{
Research partly sponsored by the military medical research funds (\#09MA016).
}

\section{REFERENCES}

Burch PA, Breen JK, Buckner JC, Gastineau DA, et al. (2000). Priming tissue-specific cellular immunity in a phase I trial of autologous dendritic cells for prostate cancer. Clin. Cancer Res. 6: 2175-2182.

Cao XT (2003). Dendritic cells and immunotherapy of liver cancer. Zhonghua Gan Zang Bing Za Zhi. 11: 133-134.

Fujii S, Shimizu K, Fujimoto K, Kiyokawa T, et al. (1999). Analysis of a chronic myelogenous leukemia patient vaccinated with leukemic dendritic cells following autologous peripheral blood stem cell transplantation. Jpn. J. Cancer Res. 90: 1117-1129.

Geiger J, Hutchinson R, Hohenkirk L, McKenna E, et al. (2000). Treatment of solid tumours in children with tumour-lysatepulsed dendritic cells. Lancet 356: 1163-1165.

Gritzapis AD, Dimitroulopoulos D, Paraskevas E, Baxevanis CN, et al. (2002). Large-scale expansion of CD3(+)CD56(+) lymphocytes capable of lysing autologous tumor cells with cytokine-rich supernatants. Cancer Immunol. Immunother. 51: 440-448.

Linn YC and Hui KM (2003). Cytokine-induced killer cells: NK-like T cells with cytotolytic specificity against leukemia. Leuk. Lymphoma 44: 1457-1462.

Maciejewski JP and Haferlach T (2012). Introduction: molecular pathogenesis of hematologic malignancies. Semin. Oncol. 39: 9-12. Märten A, Renoth S, von Lilienfeld-Toal M, Buttgereit P, et al. (2001a). Enhanced lytic activity of cytokine-induced killer cells against multiple myeloma cells after co-culture with idiotype-pulsed dendritic cells. Haematologica 86: 1029-1037.

Märten A, Ziske C, Schöttker B, Renoth S, et al. (2001b). Interactions between dendritic cells and cytokine-induced killer cells lead to an activation of both populations. J. Immunother. 24: 502-510.

Nagaraj S, Ziske C and Schmidt-Wolf IG (2004). Human cytokine-induced killer cells have enhanced in vitro cytolytic activity via non-viral interleukin-2 gene transfer. Genet. Vaccines Ther. 2: 12.

Rieser C, Ramoner R, Höltl L, Rogatsch H, et al. (1999). Mature dendritic cells induce T-helper type-1-dominant immune responses in patients with metastatic renal cell carcinoma. Urol. Int. 63: 151-159.

Stamatović D, Balint B, Tukić L, Elez M, et al. (2011). Impact of stem cell source on allogeneic stem cell transplantation outcome in hematological malignancies. Vojnosanit. Pregl. 68: 1026-1032.

Toungouz M, Libin M, Bulté F, Faid L, et al. (2001). Transient expansion of peptide-specific lymphocytes producing IFN-gamma after vaccination with dendritic cells pulsed with MAGE peptides in patients with mage-A1/A3-positive tumors. J. Leukoc. Biol. 69: 937-943.

van den Broeke LT, Daschbach E, Thomas EK, Andringa G, et al. (2003). Dendritic cell-induced activation of adaptive and innate antitumor immunity. J. Immunol. 171: 5842-5852.

Yamaguchi Y, Ohshita A, Kawabuchi Y, Ohta K, et al. (2003). Adoptive immunotherapy of cancer using activated autologous lymphocytes-current status and new strategies. Hum. Cell 16: 183-189. 